\title{
Myocardial dysfunction in patients infected with HIV: prevalence and risk factors
}

\author{
A J Jacob, G R Sutherland, A G Bird, R P Brettle, C A Ludlam, A McMillan, N A Boon
}

Abstract

Objectives-To determine the prevalence of and risk factors for myocardial dysfunction in HIV infection.

Subjects-173 patients infected with HIV underwent echocardiography. 119 were current or previous injection drug users, 38 were homosexuals, 10 were haemophiliac patients, and six were heterosexual.

Main outcome measure-Detection of impaired ventricular function.

Results-26 patients with abnormalities of ventricular size or function or both were identified. The abnormality was (a) dilated cardiomyopathy in 13 patients (eight homosexuals, three drug users, and two haemophiliacs) with a mean CD4 count of 38 cells $/ \mathrm{mm}^{3}$, which accords with end-stage disease (in addition, three patients were identified as having borderline impairment of left ventricular function); (b) left ventricular dilatation without loss of function in a further six patients; and (c) isolated right ventricular dilation in seven patients. Follow up echocardiograms were obtained in 71 patients, 18 of whom had myocardial dysfunction (103 echocardiograms, mean (SD) $2.5(0.6)$ scans per patient, mean interval 200 (116) days, range 14-538 days). These showed that in four cases of isolated right ventricular dilatation, one of isolated left ventricular dilatation, and two with borderline left ventricular dysfunction myocardial function subsequently reverted to normal. There was no excess of exposure to zidovudine in the patients with myocardial dysfunction. Similarly, patients with myocardial dysfunction had no serological evidence of excess secondary infection with Toxoplasma gondii and cytomegalovirus.

Conclusions-There was a high prevalence and wide range of myocardial dysfunction in HIV positive patients. Dilated cardiomyopathy was a feature of advanced HIV disease and affected all major risk groups for HIV infection. In contrast, isolated dilatation of either ventricle occurred at an earlier stage of HIV infection and, particularly in the case of the right ventricle, often was transient. Neither treatment with zidovudine nor infection with Toxoplasma gondii or cytomegalovirus seemed to be responsible for these findings.

(Br Heart J 1992;68:549-53)
The effects of the human immunodeficiency virus (HIV) are protean and all major organs are potential targets for infection. Cardiac involvement in HIV disease includes pericardial effusion (a common index condition in Africa), infiltration by lymphoma and Kaposi's sarcoma, and infective endocarditis, which is usually associated with injection drug use. ${ }^{1}$ Isolated case reports and small series dominated by one or other risk group have shown that HIV is also associated with heart failure secondary to myocarditis and dilated cardiomyopathy. ${ }^{2-5}$ These two conditions may represent a continuum with myocarditis progressing to cardiomyopathy in some patients. There are many potential causes of myocarditis in HIV disease including HIV itself $^{6}$ and infection with other organisms such as Toxoplasma gondii and cytomegalovirus. ${ }^{1}$ Myocardial dysfunction in HIV infection could also be an effect of zidovudine, a drug now commonly prescribed for HIV positive patients, which causes myopathy of skeletal muscle secondary to impaired replication of mitochondrial DNA. ${ }^{7}$

To date there has been no definitive assessment of the natural history and associations of cardiac dysfunction in the entire range of adult groups infected with HIV. We therefore examined 173 patients drawn from all the major risk groups for HIV infection in a prospective echocardiographic study whose principal aims were to establish the prevalence of myocardial dysfunction and determine its association with variables such as the extent of HIV disease, opportunistic infection, and adjuvant therapy.

\section{Patients and methods}

Over an 18 month period, $173 \mathrm{HIV}$ positive patients drawn from all the current major United Kingdom risk groups for HIV infection-38 homosexuals, 119 injection drug users, 10 haemophiliacs, and six heterosexuals-were examined echocardiographically on one or more occasions. Hewlett Packard Sonos 100 and 1000 machines with 2.5 and $3.5 \mathrm{MHz}$ transducers were used to measure left ventricular end diastolic and end systolic size. On-screen callipers were used to make these measurements from $M$ mode tracings derived from a short axis view at the level of the papillary muscles of the mitral valve with the patients in the left lateral position. Fractional shortening was calculated as the difference between the two diameters divided by the end diastolic value. All the echocardiographic data were stored on videotape for subsequent detailed analysis by three
Royal Infirmary, Lauriston Place, Edinburgh EH3 9YW.

Accepted for publication 20 July 1992 
independent observers including the operator. Whereas the operator was necessarily aware of the clinical history of the patients, the other two observers reported the echocardiograms independently of the operator, and were unaware both of the clinical history and the previous ultrasound result.

\section{DEFINITIONS}

Dilated cardiomyopathy-A fractional shortening of $<28 \%$ and global left ventricular hypokinesia reported by all three independent observers.

Borderline left ventricular dysfunction-A fractional shortening of $<28 \%$ and global left ventricular hypokinesia reported by only two of the three independent observers.

Isolated left ventricular dilatation-Left ventricular end diastolic diameter $>58 \mathrm{~mm}$ with a fractional shortening $>28 \%$.

Isolated right ventricular dilatation-Right ventricle equal in size or larger than the left ventricle on cross sectional views.

In 22 patients precise $M$ mode measurements could not be obtained and assessment was made exclusively on the cross sectional views. Three of these patients were classified as having cardiac dysfunction (two dilated cardiomyopathy, one isolated right ventricular dilation) and 19 were thought to be normal.

We calculated the total exposure to zidovudine in each patient by multiplying the daily dose by the number of days for which that dose had been prescribed. Blood was obtained at the time of the ultrasound study in approximately $80 \%$ of patients. Because CD4 counts were checked regularly in most patients, it was possible to obtain blood within one month of the ultrasound study in the remaining patients. Similarly, stored serum from blood taken at or shortly after the ultrasound examination was analysed for evidence of infection with Toxoplasma gondii and cytomegalovirus. A toxoplasma dye test was performed if the preliminary Toxoplasma gondii screen was positive. When this was greater than $250 \mathrm{IU} / \mathrm{ml}, \mathrm{IgM}$ was measured to establish whether the infection was recent. A cytomegalovirus titre of between 1 in 4 and 1 in 32 inclusive was interpreted as evidence of past infection. In patients with titres of more than 1 in 32 , IgM was measured to determine whether the infection was primary or recurrent. We planned to undertake repeat echocardiograms in all patients every six months, or sooner where clinically indicated. More frequent studies would have been impossible in a population consisting mainly of injection drug users who often had to be coaxed into the ultrasound suite and to remain still for the examination. Furthermore, there were potential ethical objections to performing large numbers of studies on patients whom many felt were being over investigated already. In the event, of the 173 patients recruited, 71 underwent further echocardiography. Thirty six patients died before their repeat study, 53 did not return for follow up or attended clinics outside Edinburgh, and 13 had their first echocardiogram less than six months before the end of the study period.
Results in the text are given as the mean (SD). $\chi^{2}$ and Mann-Whitney $U$ tests were used to compare different subgroups of patients.

\section{Results}

Table 1 shows the clinical and haematological characteristics of all 173 patients. The mean CD4 count of the homosexuals, which was significantly less than that of all the other risk groups, indicated more advanced disease. This is compatible with homosexuals being the first group to acquire HIV infection in the early 1980's.

Twenty nine patients (nine homosexuals, 18 drug users, and two haemophiliacs) had evidence of myocardial dysfunction on their first ultrasound study (table 2). The remaining 144 patients had structurally normal hearts.

ECHOCARDIOGRAPHIC ABNORMALITIES OF VENTRICULAR SIZE AND FUNCTION (TABLE 2)

Dilated cardiomyopathy was found in 13 patients (eight homosexuals, three drug users, and two with haemophilia) all of whom had advanced HIV disease (CD4 count 38 (55) cells $/ \mathrm{mm}^{3}$ ) (table 2). Mean fractional shortening for this group was $20.9(4.7) \%$ (range $14-27 \%)$. There was a significant excess of homosexuals in this group $(21.1 \%$ affected $v$ $2.5 \%$ of injection drug users affected, p $<0.001$ ), probably because the homosexual patients had more advanced HIV disease than the drug users. At least seven of these patients had symptoms and signs of heart failure including fatigue, breathlessness, tachycardia, ankle oedema, gallop rhythm, and pulmonary crackles. Before echocardiographic examination only three patients were thought to have a cardiac disorder. Five patients had repeat echocardiography $(2.2(0.5)$ echocardiograms per patient, mean interval 184 (154) days, range 49-476 days) and in every case there was evidence of persistent global impairment of left ventricular function (fig 1).

Borderline left ventricular dysfunctionThere were three patients in this group. Their fractional shortening was $26(1 \cdot 7) \%$ (range 24 $27 \%$ ). All three had symptoms of but no signs consistent with impaired left ventricular function. Two patients underwent repeat echocardiography, one five months later and the other on two occasions five and six months later. In both patients ventricular function had reverted to normal (fig 1). One of these patients was subsequently shown to have had Pneumocystis carinii pneumonia at the time of his initial echocardiogram.

Isolated left ventricular dilatation-Six patients had left ventricular dilation without loss of function. Their left ventricular end diastolic diameter was $60.7(1.0) \mathrm{mm}$ (range 59-62 $\mathrm{mm}$ ) and their fractional shortening was normal at $36.5(3.8) \%$ (range $31-41 \%$ ). There was no significant difference in fractional shortening compared with patients with normal hearts. Repeat echocardiography was performed in four of the six patients in this group $(2.3(0.5)$ echocardiograms per patient, mean interval 263 (149) days, range 171-523 days). 
Table 1 Clinical profile of 173 patients with HIV infection

\begin{tabular}{|c|c|c|c|c|}
\hline Risk group & $M$ & $F$ & $\begin{array}{l}\text { Age (mean }(S D)) \\
\text { (range) }(y r)\end{array}$ & $\begin{array}{l}C D 4^{\star}(\text { mean }(S D)) \\
\text { (range }\left(\text { cells } / \mathrm{mm}^{3}\right)\end{array}$ \\
\hline $\begin{array}{l}\text { Injection drug users }(n=119) \\
\text { Homosexual }(n=38) \\
\text { Haemophiliac }(n=10) \\
\text { Heterosexual }(n=6)\end{array}$ & $\begin{array}{r}79 \\
38 \\
10 \\
3\end{array}$ & $\frac{40}{3}$ & $\begin{array}{l}29.6(4.8)(22-44) \\
35.9(7.9)(26-59) \\
37.9(9.0)(28-56) \\
43.8(15 \cdot 0)(29-63)\end{array}$ & $\begin{array}{r}178(189) \dagger(0-931) \\
72(104) \dagger(0-388) \\
108(121)(10-390) \\
128(100)(3-243)\end{array}$ \\
\hline
\end{tabular}

*Normal range for CD4 is $500-1500$ cells $/ \mathrm{mm}^{3}$.

tp $<0.0001$.
In one patient ventricular function reverted to normal and three had persistent isolated left ventricular dilatation (fig 1 ). In this subgroup, there were no significant changes either in left ventricular end diastolic diameter $(60 \cdot 7$ $(0.6) \mathrm{mm}$ to $60.3(1.2) \mathrm{mm}$ or in fractional shortening $(34.0(6 \cdot 6) \%$ to $36 \cdot 7(3.2) \%)$.

Isolated right ventricular dilatation-Seven patients were found to have right ventricular dilatation with normal left ventricular size and function. Their fractional shortening was normal at $37 \cdot 2(7 \cdot 7) \%$ (range $28-51 \%$ ). One patient had a large pericardial effusion and clinical and Doppler evidence of pulmonary hypertension. A subsequent perfusion lung scan indicated a high probability of multiple pulmonary emboli and treatment with warfarin was started. All seven underwent repeat echocardiography $(2.6(0.5)$ echocardiograms per patient, mean interval 240 (100) days, range 104-400 days). In four of these patients ventricular function subsequently became normal and three of them had had a chest infection at the time of their initial assessment (fig 1). Of the remaining three patients with persistent right ventricular dilatation one had pulmonary hypertension and another tricuspid incompetence due to previous endocarditis.

\section{ADDITIONAL OBSERVATIONS}

Fifty three patients with normal hearts subsequently had repeat echocardiography (78 echocardiograms, $2.5(0.6)$ scans per patient, mean interval 193 (115) days) and in two dilated cardiomyopathy had developed (fig 1). Both these patients had late stage HIV disease and there was no evidence of recent acute secondary infection to account for the considerable decline in left ventricular function. Pericardial effusions were rare, being found in just four patients (one had isolated right ventricular dilatation and multiple pulmonary emboli, two had dilated cardiomyopathy, and the fourth had normal ventricles). Just one patient had active infective endocarditis affecting the tricuspid valve.

TREATMENT WITH ZIDOVUDINE (TABLE 2)

Dilated cardiomyopathy-All but one of these patients had been treated with zidovudine. However, neither the proportion of patients treated nor their mean total dose was significantly different from those patients without myocardial dysfunction.

Borderline left ventricular dysfunction-All three patients had received zidovudine but their mean total dose was not significantly different from those patients with structurally normal hearts.

Isolated left ventricular dilatation-Four of the six patients had undergone treatment with zidovudine, but again exposure was not significantly different from that in any other group.

Isolated right ventricular dilatation-Though the proportion of patients treated with zidovudine $(6 / 7)$ was similar to all the other groups, the mean total dose $(546.7(246.3) \mathrm{g}$ was significantly greater than that received by patients with dilated cardiomyopathy $(p<0.05)$ and those with structurally normal hearts $(\mathrm{p}<0.005)$.

\section{SECONDARY INFECTION WITH TOXOPLASMA \\ GONDII AND CYTOMEGALOVIRUS}

Of the 29 patients with cardiac abnormalities, 25 had never been infected with Toxoplasma gondii, two had evidence of past infection, and the status of two was unknown. Similarly, seven of these patients had never been infected with cytomegalovirus, three had experienced recurrent infection around the time of their echocardiogram, 16 had had an infection in the past, and the status of three was unknown. Infection with Toxoplasma gondii or cytomegalovirus was not significantly more common in patients with myocardial dysfunction than in patients with normal hearts (fig 2).

Serum was available from 11 of the 13 patients with dilated cardiomyopathy. Nine showed no evidence of exposure to Toxoplasma gondii and two had been infected in the past. Similarly, three patients had never been infected with cytomegalovirus and eight had evidence of past infection. Again, it was apparent that there was no excess secondary infection by these organisms in this group of patients.

\section{Discussion}

We have established that myocardial dysfunction in HIV positive subjects is common and affects all the major risk groups for HIV infection in the United Kingdom. In this series, dilated cardiomyopathy occurred late in the course of the disease whereas isolated left and right ventricular dilatation were found, sometimes transiently, at an earlier stage of

Table 2 Characteristics of patients with heart muscle disease and those without

\begin{tabular}{|c|c|c|c|c|c|c|c|}
\hline & \multirow[b]{2}{*}{$\begin{array}{l}\text { Homo- } \\
\text { sexual (38) }\end{array}$} & \multirow[b]{2}{*}{$\begin{array}{l}\text { Drug } \\
\text { users (119) }\end{array}$} & \multirow[b]{2}{*}{$\begin{array}{l}\text { Haemo- } \\
\text { philiac (10) }\end{array}$} & \multirow[b]{2}{*}{$\begin{array}{l}\text { Hetero- } \\
\text { sexual (6) }\end{array}$} & \multirow[b]{2}{*}{ 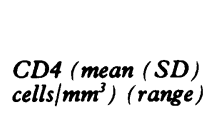 } & \multicolumn{2}{|c|}{ Treatment with zidovudine } \\
\hline & & & & & & $\begin{array}{l}\text { Number } \\
(\%)\end{array}$ & $\begin{array}{l}\text { Total dose } \\
(\text { mean }(S D) g) \text { (range) }\end{array}$ \\
\hline $\begin{array}{l}\text { Cardiomyopathy }(n=13) \\
\text { Borderline }(n=3) \\
\text { LV dilatation }(n=6) \\
\text { RV dilatation }(n=7) \\
\text { Normal }(n=144)\end{array}$ & $\begin{array}{r}8 \\
1 \\
0 \\
0 \\
29\end{array}$ & $\begin{array}{r}3 \\
2 \\
6 \\
7 \\
101\end{array}$ & $\begin{array}{l}2 \\
0 \\
0 \\
0 \\
8\end{array}$ & $\begin{array}{l}0 \\
0 \\
0 \\
0 \\
6\end{array}$ & $\begin{array}{l}38(55) \star(0-145) \\
81(64)(13-139) \\
215(299)(4-783) \\
105(105)(4-266) \\
160(175)(0-931)\end{array}$ & $\begin{array}{c}12(92) \\
3(100) \\
4(67) \\
6(86) \\
109(76)\end{array}$ & $\begin{array}{l}285 \cdot 4(245 \cdot 8)(23 \cdot 1-880 \cdot 9) \\
525 \cdot 9(392 \cdot 2)(215-966 \cdot 6) \\
472 \cdot 2(234 \cdot 0)(136-626) \\
546 \cdot 7(246 \cdot 3)^{\star \star}(220 \cdot 2-824 \cdot 2) \\
236 \cdot 3(334 \cdot 3)(0 \cdot 3-2500)\end{array}$ \\
\hline
\end{tabular}

${ }^{\star} \mathrm{p}<0.02 v$ normal; ${ }^{\star \star} \mathrm{p}<0.005 v$ normal and $\mathrm{p}<0.05 v$ cardiomyopathy 


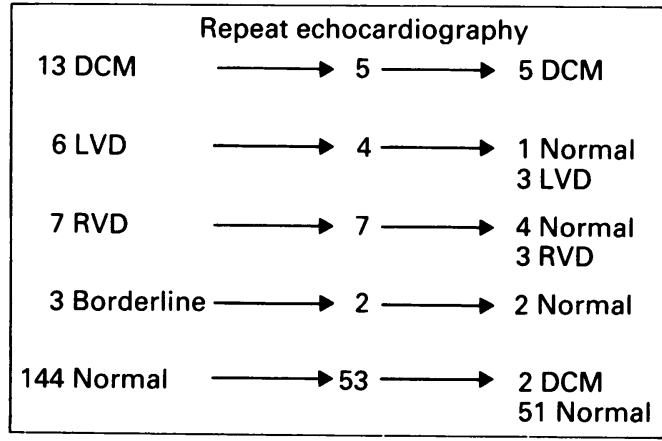

Figure 1 HIV heart muscle disease at follow up echocardiographic examination. DCM, dilated cardiomyopathy; LVD, isolated left ventricular dilatation; $R V D$, isolated right ventricular dilatation, borderline, borderline left ventricular dysfunction. Intervals (mean (SD)) between echocardiograms for each group are as follows: $D C M=184$ (154) days, $L V D=263$ (149) days; $R V D=240$ (100) days borderline $=153$ (13) days; normal $=193$ (115) days

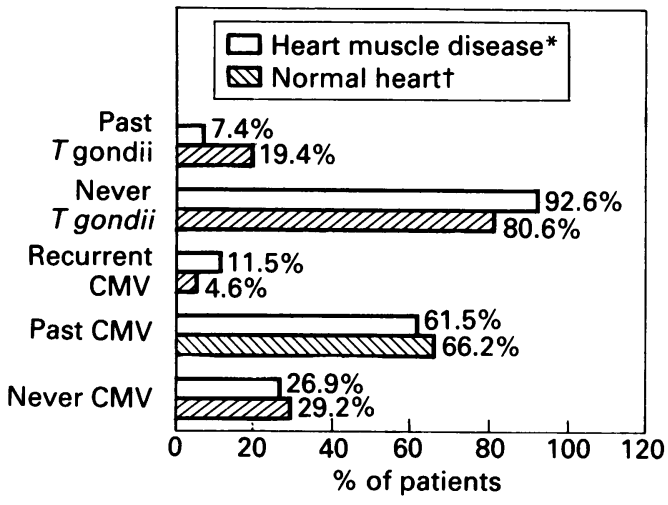

Figure 2 Information on infection with Toxoplasma gondii and cytomegalovirus in patients with HIV heart muscle disease and in patients with normal hearts. These results were derived from serological techniques.

*In two patients $T$ gondii status was unknown and in three patients CMV status was unknown.

$\dagger$ In 46 T gondii status was unknown and in $79 \mathrm{CMV}$ status was unknown.

HIV infection and exclusively in injection drug users.

Deaths caused by opportunistic infection in HIV patients are declining and the median survival time from the diagnosis of AIDS is increasing. ${ }^{8}$ This suggests that more patients will survive to develop the complications of late stage HIV disease including dilated cardiomyopathy.

Only some of the patients with dilated cardiomyopathy in our series had symptoms and signs consistent with impaired left ventricular function. In addition, before echocardiography less than $25 \%$ of this group was thought to have a cardiac disorder. Similar findings have been reported in an American series in which myocardial dysfunction was assessed in a small group of HIV positive children and these findings reflect the tendency to ascribe symptoms and signs of heart failure to non-cardiac causes. $^{9}$

The cause of dilated cardiomyopathy in HIV infection is unknown. It may occur as a result of HIV itself acting either directly ${ }^{6}$ or indirectly via immunological mechanisms. ${ }^{10}$ Secondary infection with opportunistic organisms may also be important, particularly Toxoplasma gondii and cytomegalovirus which occur commonly in HIV infection and have been associated with myocarditis. ${ }^{11} 12$ Drugs used in the treatment of HIV disease and its complications can have adverse cardiac effects. Zidovudine can cause a skeletal myopathy secondary to inhibition of mitochondrial DNA polymerase ${ }^{7}$ and both pentamidine and ganciclovir can predispose patients to ventricular tachycardia. ${ }^{13} 14$ Malnutrition has been reported in patients with HIV infection. ${ }^{15}$ Selenium deficiency is thought to be responsible for a naturally occurring form of dilated cardiomyopathy in China (Keshan disease), ${ }^{16}$ and it has also been associated with cardiac dysfunction in HIV infection. ${ }^{17}$ Finally, cytokines such as tumour necrosis factor, have been found in high concentrations in HIV patients and these intercellular messengers may be responsible for some of the end-organ damage caused by the virus. ${ }^{18}$ The aetiology of myocardial dysfunction in HIV infection is probably complex and multifactorial.

In our study we found no serological evidence either of recent infection with Toxoplasma gondii and cytomegalovirus or an excess of past infection with these organisms in the patients with myocardial dysfunction. Furthermore, separate analysis of the patients with dilated cardiomyopathy showed that some had never been infected by either organism. This militates against a potential pathogenetic role for these agents. However, it is possible that the results of serological tests are misleading in the context of the immune paresis associated with advanced HIV disease. Further work with the polymerase chain reaction, in-situ hybridisation, and immunohistochemical and culture techniques on endomyocardial biopsy and post-mortem material is required.

This is the first major study to show that treatment with zidovudine does not predispose towards cardiac dysfunction. Although most patients with myocardial dysfunction had been treated with zidovudine, neither the proportion of patients treated nor the mean total dose in each group was significantly different from the results in patients with structurally normal hearts. It is not clear why the group with isolated right ventricular dilatation had received a greater mean total dose of zidovudine than those with dilated cardiomyopathy and those with normal hearts. One possible explanation is that patients who have been in hospital with respiratory infections, and who were therefore more at risk of isolated right ventricular dilatation developing (see below), are also more likely to be treated with zidovudine than those remaining well in the community. However, in all the heart muscle disease groups some patients (except those described as having "borderline left ventricular dysfunction") had never received zidovudine. Moreover, in several patients with isolated right ventricular dilatation ventricular function subsequently reverted to normal while the patient continued to be treated with this drug. Taken together, these findings provide strong circumstantial evidence that zidovudine is not implicated in the development of myocardial dysfunction in HIV infection. It was not possible to determine whether all patients were taking their zidovudine as prescribed. 
However, mean corpuscular volume was increased (a feature commonly associated with zidovudine treatment $\left.{ }^{19}\right)$ in most $(100 / 118$ $(84.7 \%))$ in patients who were prescribed zidovudine and, in addition, recognised side effects occurred in some patients. On balance, it is unlikely that compliance was different either in the various heart muscle disease groups or in those without cardiac dysfunction.

This study confirms the findings of Blanchard et al who showed that left ventricular function can be transiently impaired, and that the presence of left ventricular dysfunction on a single echocardiogram (the borderline group in our study) does not necessarily imply a poor prognosis. ${ }^{20}$

The significance of isolated left ventricular dilatation is unclear but it may represent an early stage in the development of dilated cardiomyopathy, despite the fact that in our series further echocardiograms did not show any significant change in left ventricular size or deterioration of function. The aetiology of isolated right ventricular dilatation is likely to be multifactorial. It may arise as part of a generalised myopathic process, from pulmonary hypertension due to pulmonary emboli or recurrent chest infections, ${ }^{121}$ from volume overload secondary to tricuspid incompetence mediated by endocarditic valve damage, or from acute respiratory infection. In our series two of the three patients with persistent isolated right ventricular dilatation had either clinical evidence of pulmonary hypertension or a history of prior infective endocarditis of the tricuspid valve with Doppler evidence of significant tricuspid incompetence. Furthermore, three of the four patients with isolated right ventricular dilatation in whom ventricular function subsequently reverted to normal had a chest infection at the time of their initial ultrasound assessment. These findings suggest that many cases of isolated right ventricular dilatation are related to pressure/volume overload of the right ventricle or acute respiratory tract infection rather than a myopathic process.

In conclusion, myocardial dysfunction in HIV infection is common, takes various forms, and affects all major risk groups. Dilated cardiomyopathy occurs late in the course of HIV infection and is often associated with symptoms and signs that may be attributed mistakenly to other disease processes. Transient impairment of left ventricular function can also occur, possibly in relation to intercurrent infection. Similarly, isolated right ventricular dilatation is often associated with acute respiratory infection and therefore can also be transient. Persistence of dilatation may be related to pressure or volume overload of the right ventricle. Cardiac dysfunction does not seem to be related to treatment with zidovudine or infection with Toxoplasma gondii and cytomegalovirus. A direct effect of the virus itself remains a strong possibility. This possibility requires further research.

It is likely that better treatment of opportunistic infections and of HIV disease will result in more patients surviving to develop malignancies and end-organ failure. ${ }^{822} \mathrm{We}$ believe that heart failure due to myocardial dysfunction will become an important cause of morbidity and mortality in this group. Prompt recognition and treatment is important because palliative therapy with diuretics and vasodilators can be worthwhile.

We thank Dr J Inglis and Dr H Cubie of the Regional Virus Laboratory for performing the serological analyses, Mrs Marjorie Boubert for help with the echocardiography and Mrs Jean Cunningham for expert secretarial assistance.

A $\mathbf{J} \mathbf{J}$ is supported by a Junior Research Fellowship from the British Heart Foundation.

1 Acierno LJ. Cardiac complications in acquired immunodeficiency syndrome (AIDS): A review. J Am Coll Cardio 1989;13:1144-54

2 Anderson DW, Virmani R, Reilly JM, et al. Prevalen myocarditis at necropsy in the acquired immunodeficiency syndrome. J Am Coll Cardiol 1988;11:792-9.

3 Reilly JM, Cunnion RE, Anderson DW, et al. Frequency of myocarditis, left ventricular dysfunction and ventricula tachycardia in the acquired immune deficiency syndrome. Am J Cardiol 1988;62:789-93.

4 Corallo S, Mutinelli MR, Moroni M, et al. Echocardiography detects myocardial damage in AIDS: prospective graphy detects myocardial damage in AIDS: pros

5 Himelman RB, Chung WS, Chernoff DN, Schiller NB, Hollander $\mathrm{H}$. Cardiac manifestations of human immunodeficiency virus infection: a two-dimensional echodeficiency virus infection: a two-dimensional echo-

6 Calabrese LH, Proffitt MR, Yen-Lieberman B, et al. Congestive cardiomyopathy and illness related to the acquired gestive cardiomyopathy and illness related to the acquired
immunodeficiency syndrome (AIDS) associated with immunodeficiency syndrome (AIDS) associated with isolation of retroviru

7 Arnaudo E, Dalakas M, Shanske S, Moraes CT, DiMauro S, Schon EA. Depletion of muscle mitochondrial DNA in AIDS patients with zidovudine-induced myopathy Lancet 1991;337:508-10.

8 Peters BS, Beck EJ, Coleman DG, et al. Changing disease patterns in patients with AIDS in a referral centre in the United Kingdom: the changing face of AIDS. Br Med 1991;302:203-7.

9 Lipshultz SE, Chanock S, Sanders SP, Colan SD, PerezAtayde A, McIntosh K. Cardiovascular manifestations of human immunodeficiency virus infection in infants and human immunodeficiency virus infection

10 Ho DD, Pomerantz RJ, Kaplan JC. Pathogenesis of infection with human immunodeficiency virus. $N$ Engl J Med tion with human

11 Vynn Adair O, Randive N, Krasnow N. Isolated toxoplasma myocarditis in acquired immune deficiency syndrome. $A m$ Heart $J$ 1989;118:856-7.

12 Lafont A, Marche C, Wolff M, et al. Myocarditis in acquired immunodeficiency syndrome (AIDS): etiology and prog nosis [abstract]. J Am Coll Cardiol 1988;11:196.

13 Wharton JM, Demopulos PA, Goldschlager N. Torsade de pointes during administration of pentamidine isethionate. Am J Med 1987;83:571-6.

14 Cohen AJ, Weiser B, Afzal Q, Fuhrer J. Ventricular tachycardia in two patients with AIDS receiving ganciclovir (DHPG). AIDS 1990;4:807-9.

15 Kotler DP. Malnutrition in HIV infection and AIDS. AIDS 1989;3(suppl 1):S175-S180.

16 Keshan Disease Research Group (Chinese Academy of Medical Sciences). Observations on effect of sodium selenite in the prevention of Keshan disease. Chin Med J 1979;92:471-6.

17 Zazzo JF, Chalas J, Lafont A, Camus F, Chappuis P. Is nonobstructive cardiomyopathy in AIDS a selenium nonobstructive cardiomyopathy in A En a selenium 12:537-8.

18 Matsuyama T, Kobayashi N, Yamamoto N. Cytokines and HIV infection: is AIDS a tumor necrosis factor disease? HIV infection: is AIDS

19 Richman DD, Fischl MA, Grieco MH, et al. The toxicity of azidothymidine (AZT) in the treatment of patients with AIDS and AIDS-related complex. A double-blind, placebo-controlled trial. N Engl J Med 1987;317:192-7.

20 Blanchard DG, Hagenhoff C, Chow LC, McCann HA, Dittrich HC. Reversibility of cardiac abnormalities in human immunodeficiency virus (HIV)-infected individuals: a serial echocardiographic study. J Am Coll Cardiol 1991;17:1270-6.

21 Himelman RB, Dohrmann M, Goodman P, et al. Severe pulmonary hypertension and cor pulmonale in th acquired immunodeficiency syndrome. Am J Cardio 1989;64:1396-9.

22 Coplan NL, Bruno MS. Acquired immunodeficiency syndrome and heart disease: the present and the future. $A m$ Heart $J 1989 ; 117: 1175-7$ 